\title{
Subluxación primaria recidivante medial de rótulas. Revisión a largo plazo de un caso asociado a síndrome de desalineación torsional severa
}

\author{
Primary recurrent medial subdislocation of both patellae. Long-term \\ review of an exceptional case of miserably malalignment syndrome
}

\author{
S. García-Mata, A. Hidalgo-Ovejero
}

\section{RESUMEN}

Presentamos una revisión a largo plazo de una chica de 11 años de edad con subluxación medial recidivante de ambas rótulas de varios meses de evolución asociado a síndrome de desalineación torsional severa. No ha sido descrito ningún caso de luxación o subluxación medial de rótulas hasta el momento.

Había sufrido luxación medial de la rótula derecha tres años antes tras un salto, reducida por ella misma y no inmovilizada, seguida de 10 episodios de subluxación y tres de luxaciones mediales. Acudió a consulta por imposibilidad a la deambulación por subluxación persistente o habitual y al dolor fémoro-rotuliano, principalmente en la rodilla derecha.

Presentaba subluxación medial habitual de rótulas, con desplazamiento rotuliano medial, amiotrofia cuadricipital, inestabilidad medial evidente de ambas rótulas. El test de supresión medial era positivo. Mostraba anteversión femoral excesiva de caderas (RI: $90^{\circ}$, RE: $30^{\circ}$ ), genu varum, torsión tibial neutra, patella alta, surcos trocleares displásicos con hipoplasia del cóndilo medial y rótulas displásicas (Wiberg tipo III).

Se realizó osteotomía femoral subtrocantérea desrotatoria y realineación proximal rotuliana bilateral. Tras la cirugía recuperó la estabilidad rotuliana bilateral, marcha y carrera normales, con tests de Smillie y aprehensión (-), y movilidad de cadera de RE: $65^{\circ}$, RI: $50^{\circ}$.

Quince años tras la cirugía la paciente aquejaba dolor antero-lateral de la rodilla derecha en la deambulación prolongada, al subir y bajar escaleras y la sedestación prolongada, que se diagnosticó de síndrome de hiperpresión rotuliana externa.

Palabras clave. Luxación medial rotulas. Síndrome desalineación torsional severa. Osteotomía desrotatoria femoral.

\begin{abstract}
We present a long-term review of a girl aged 11 years and 4 months with medial primary recurrent subluxation of both patellae of several months evolution associated with miserably malalignment syndrome. Not one case of medial recurrent dislocation of the patellae has been described previously.
\end{abstract}

Three years previously following a jump she had suffered a right patellar luxation - self-reduced and not immobilised - followed by 10 subsequent episodes of subluxation and three more medial luxations. She could hardly walk autonomously due to persistent or habitual subluxation and patellofemoral pain, mainly in the right knee.

Physical examination revealed habitual medial subluxation of both patellae, with clear medial patellar displacement, quadriceps amyotrophy and medial instability. The medial subluxation suppression test was positive. She showed excessive femoral anteversion of the hips (IR: $90^{\circ}$, ER: $30^{\circ}$ ), genu varum, neutral tibial torsion, patella alta, dysplastic trochlear grooves with medial condyle hipoplasia and both patellae were dysplastic (Wiberg type III).

We performed a derotation subtrochanteric femoral osteotomy and bilateral proximal patellar realignment. Following surgery, bilateral stability of both sides permitted normal walking and running, as well as apprehension and the Smillie test (-), with a hip mobility of $65^{\circ} \mathrm{ER}$ and $50^{\circ} \mathrm{IR}$.

Fifteen years after the surgery the patient complains of antero-external knee pain in the right knee during prolonged walking, in getting up and down stairs and when in a prolonged sitting position, diagnosed as excessive lateral pressure syndrome.

Key words. Medial dislocation patellae. Miserably malalignment syndrome. Femoral osteotomy.
Servicio de Cirugía Ortopédica y Traumatología. Sección de Traumatología y Ortopedia Infantil. Hospital Virgen del Camino. Pamplona.

Fecha de recepción el día 12 de marzo de 2007 Aceptado para su publicación el 1 de abril de 2007

Aceptación definitiva el 15 de abril de 2007

\section{Correspondencia:}

Serafin García Mata

Hospital Virgen del Camino

C/ Irunlarrea, 4

31008 Pamplona

Tfno. 848422644

Fax. 848429924

E-mail: sgarcima@cfnavarra.es 


\section{INTRODUCCIÓN}

Los ejes de las EEII se desarrollan de diferentes formas dependiendo de factores genéticos, ambientales o mecánicos así como por el poder de remodelación óseo ejercido por los músculos y su actividad ${ }^{1}$.

Durante el desarrollo ocurren variaciones fisiológicas de la alineación de las EEII que han sido claramente definidas por Salenius y Vanka ${ }^{2}$, así como Cahuzac ${ }^{3}$. Normalmente la combinación de anteversión femoral severa (AFE) y torsión tibial externa (TTE) se conoce como síndrome de malaalineación o desalineación torsional severa ${ }^{4}$ ${ }^{6}$. Es bien conocido que la mayor parte de los trastornos torsionales se resuelven o acomodan con la edad. Tan sólo un pequeño porcentaje de niños mayores con trastornos cosméticos o funcionales requieren corrección quirúrgica ${ }^{5}$. La mayor parte de las inestabilidades de rótula son debidas a algún grado de desalineación en las extremidades inferiores (EEII).

Se ha demostrado que la desalineación de los ejes en las EEII puede ocasionar patología fémoro-rotuliana, especialmente dolor fémoro-rotuliano, luxaciones y subluxaciones de rótula, pero siempre se han descrito en dirección externa.

Eppley $^{7}$ acuñó el término "inward patella" (rótula hacia dentro) en referencia a las subluxaciones mediales, en comparación con aquellas en dirección superior o inferior.

Varios autores ${ }^{1,5,6}$ han descrito pacientes con trastornos rotulianos secundarios a alteraciones de la alineación de las EEII denominadas: "severe torsional malalignment syndrome" ${ }^{5}$ or "miserably malaligned syndrome" ${ }^{\prime \prime}$. Esta rara ocurrencia ha generado acuerdo en referencia a realizar osteotomía de realineación. Dichos trastornos son luxaciones recidivantes en dirección externa, pero no luxaciones recidivantes mediales como ocurría en el caso que presentamos. En este caso se producirá, secundariamente, una alteración en el estabilizador pasivo externo.

La subluxación primaria medial de la rótula es una condición excepcional que hasta el momento no ha sido descrita en la bibliografía internacional, ni en los princi- pales textos de trastornos fémoro-rotulianos $^{8}$. La inestabilidad medial secundaria o iatrogénica ha sido reconocida como una entidad nosológica diferenciada, causada por una liberación retinacular externa excesiva $^{9-12}$. Para que ocurra una inestabilidad medial debe alterarse el estabilizador rotuliano pasivo externo.

El retináculo lateral para-rotuliano es el estabilizador pasivo externo principal. Tiene dos componentes ${ }^{8}$ : 1) superficial oblícuo (va de cintilla iliotibial a rótula); 2 ) profundo transverso. Este último se compone de tres partes: porción epicóndilorotuliana o ligamento patelo-femoral lateral, retináculo profundo transverso (va de la cintilla iliotibial a rótula y es denso) que es la estructura de soporte lateral primaria de la rótula, y la banda patelofemoral o ligamento menisco-patelar.

La excepcionalidad de la subluxación recidivante primaria medial de rótulas que presentamos, secundarias a la desalineación de EEII, y de su manejo nos impulsa a mostrar su revisión a largo plazo, como objetivo del trabajo, con objeto de disponer de la información suficiente para valorarla en su evolución.

\section{CASO CLÍNICO}

Niña de 11 años y 4 meses de edad que acudió a nuestra consulta remitida de otro centro hospitalario aquejando dificultad progresiva para la deambulación autónoma de varios meses de evolución. Refería haber sufrido, tres años antes, un episodio de luxación de rótula derecha a raíz de un salto. Dicho episodio de luxación fue descrito como luxación medial tanto por la paciente como por el médico que la atendió. La luxación fue reducida por manipulación y no inmovilizada. Posteriormente ocurrieron otros 10 episodios de subluxación y otros tres más de luxación (la paciente refería que la rótula se le salía "hacia dentro de la rodilla" y "no hacia fuera"), no habiendo sido inmovilizada en ningún episodio. En el mes precedente a la consulta refería haber tenido 3 episodios de subluxaciones, tras lo cual apenas podía realizar marcha de forma autónoma por subluxación persistente y dolor. En el lado izquierdo refería tres episodios de subluxación medial en los seis últimos meses.

La paciente refería debilidad progresiva en ambas rodillas, aprensión medial rotuliana bilate- 
ral, chasquidos, dolor persistente a la marcha y severa limitación en sus actividades habituales.

El desplazamiento medial de la rótula recreaba los síntomas dolorosos. En ese momento acudió a nuestra consulta en silla de ruedas debido a la imposibilidad de deambulación autónoma, ya que fallaba con subluxación y dolor mecánico.

En la exploración física se evidenciaba subluxación medial recidivante de ambas rótulas (más evidente en la derecha), grado +2 en la derecha $\mathrm{y}+1$ en la izquierda como gradación cuantitativa de subluxación pasiva (máximo +3) (Figs. 1 y 2) con claro desplazamiento medial rotuliano, amiotrofia cuádriceps bilateral, cepillos $(+)$, clara inestabilidad medial y aprensión de ambas rótulas (Smillie +). Existía una facilidad para realizar subluxación de las

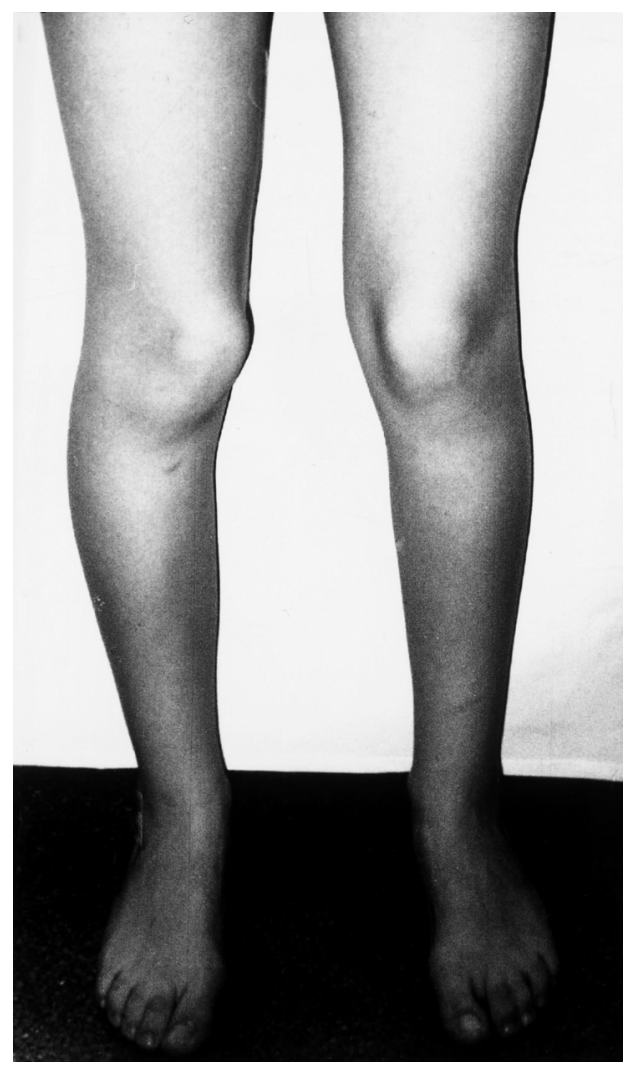

Figura 1. Subluxación habitual medial bilateral de las rótulas, más evidente en el lado derecho. rótulas en dirección medial, pero no lateral. El test de la supresión de la subluxación medial ${ }^{5}$ era (+). Las maniobras meniscales, Lachmann $\mathrm{y}$ pivot shift tests fueron negativos. La contracción isométrica del cuádriceps provocaba desplazamiento medial de la rótula. Presentaba una mayor prominencia del cóndilo externo que el interno.

La exploración de la cadera mostraba anteversión femoral excesiva (AFE) (RI: 90ㅜ, RE: 30ํ) (Figs. 3a y 3b). La exploración de la rodilla evidenciaba genu varum y torsión tibial neutra, con ángulo Q bilateral de $15^{\circ}$. En el estudio radiográfico mostraba malposición rotuliana (patella alta), con un índice de Insall-Salvati de $1,25(\mathrm{~N}: 1,04 \pm 0,11)$, desplazamiento medial rotuliano, y displasia de los surcos trocleares, con un ángulo de surco de $150^{\circ}\left(\mathrm{N}: 137^{\circ} \pm 6^{\circ}\right)$ y

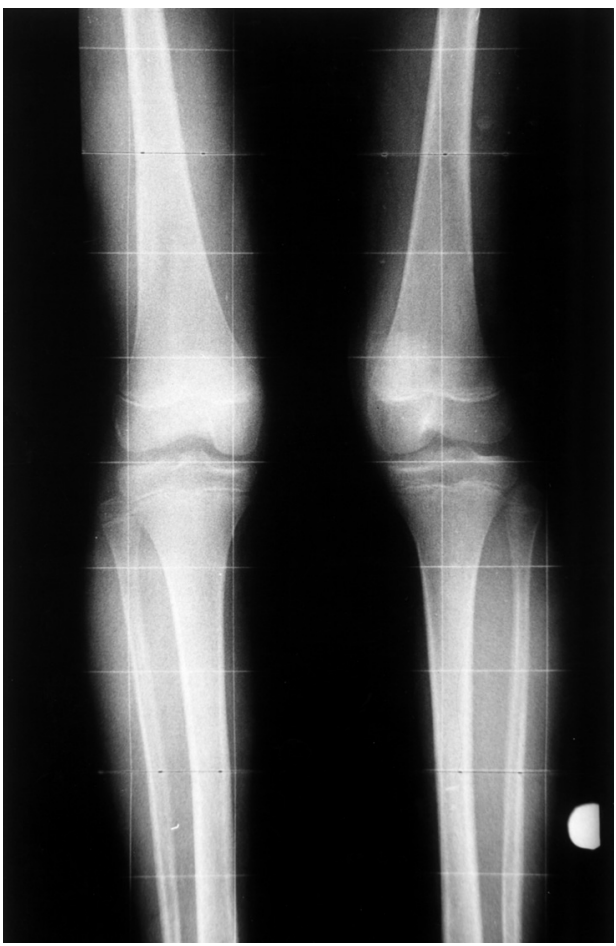

Figura 2. Teleradiografía en carga de las EEIl mostrando subluxación medial de rótulas. 

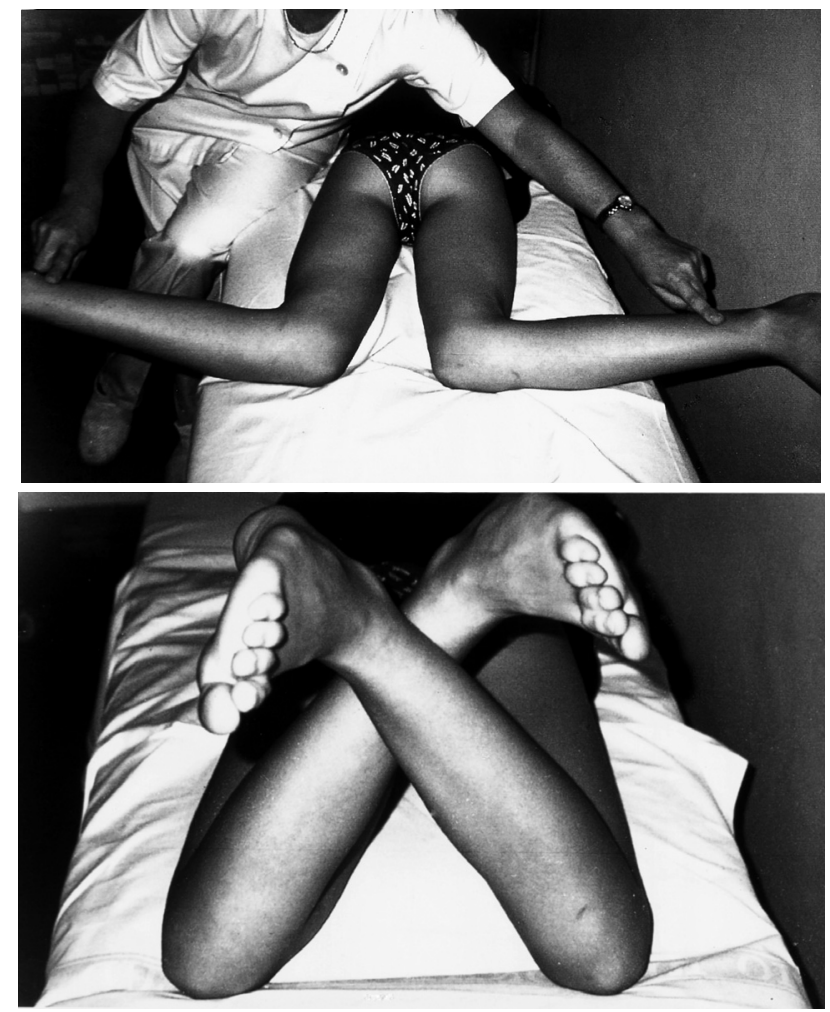

Figuras 3a y 3b. La exploración de la cadera mostraba anteversión femoral excesiva (RI: $90^{\circ}$ y RE: $\left.30^{\circ}\right)$.
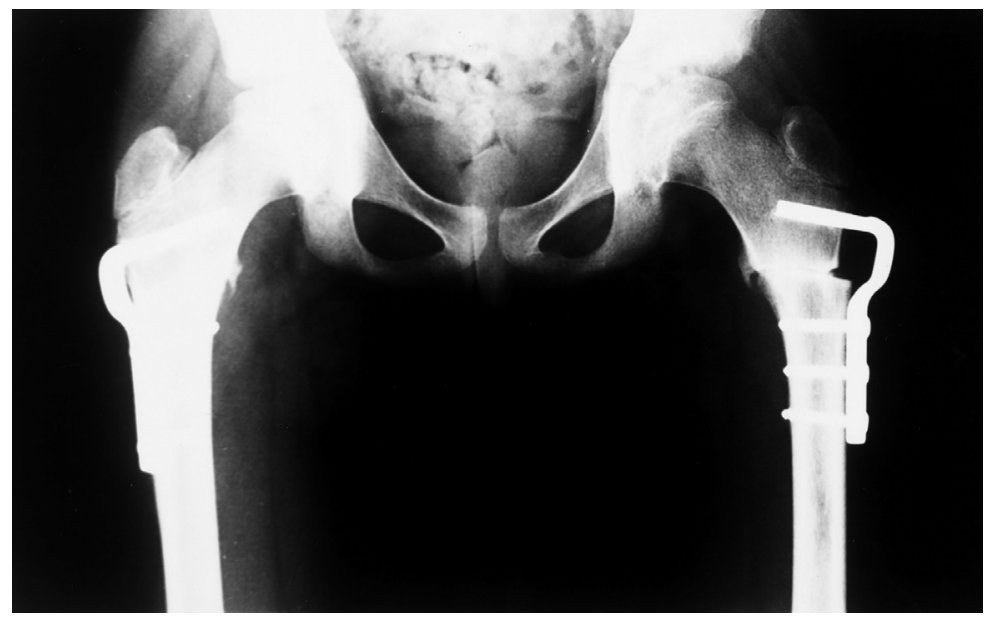

Figura 4. Tres meses tras la última intervención quirúrgica. La paciente refiere ausencia de sintomatología con estabilidad clínica objetiva de las rótulas. 
un ángulo de congruencia de $-20^{\circ}\left(\mathrm{N}:-8^{\circ} \pm 6^{\circ}\right)$. Ambas rótulas eran displásicas (Wiberg tipo III). La exploración clínica y radiológica de nuestra paciente evidenciaba el cuadro de "miserably malaligned extremities" (síndrome de desalineación severa de las EEII).

Una semana más tarde, realizamos osteoto-

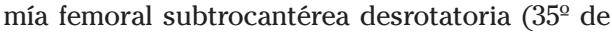
rotación externa) (Fig. 4) y realineación proximal rotuliana bilateral (apertura retináculo medial y plicatura del lateral), en dos tiempos (cada extremidad separadamente), para asegurar el recorrido y deslizamiento rotuliano centrado.

Tras la intervención y rehabilitación del lado derecho, se consiguió estabilidad clínica objetiva y subjetiva de la rótula pero la paciente no podía realizar la marcha normal por dolor e inestabilidad progresiva del lado izquierdo.

Tras realizar la cirugía del lado izquierdo se consiguió la estabilidad de ambos lados que

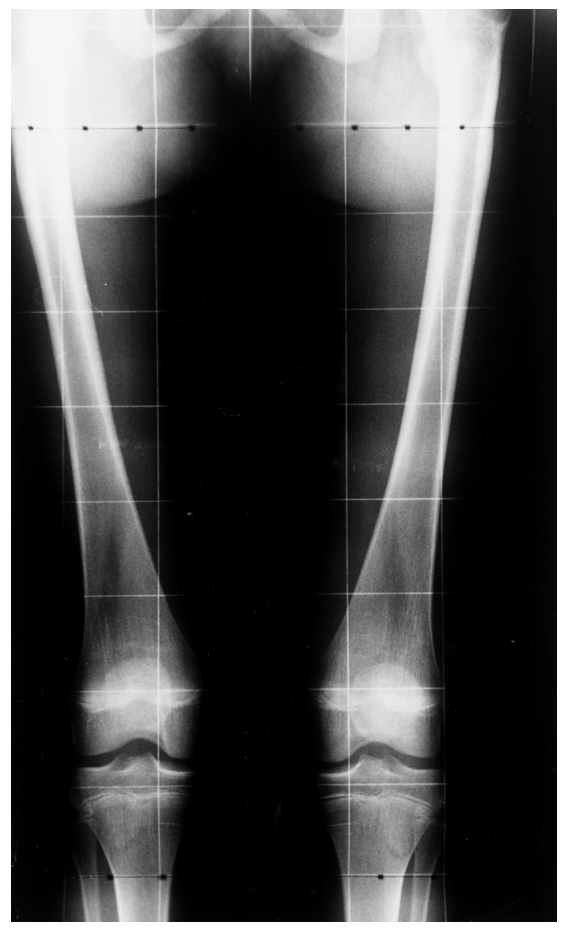

Figura 5. Teleradiografía en carga de EEII postoperatoria (seis meses más tarde) mostrando posición correcta de ambas rótulas. le permitía realizar marcha y carrera normales. El apprehension test y el test de Smillie se negativizaron, con una movilidad de $65^{\circ}$ de RE y $50^{\circ}$ de RI de ambas caderas (Figs. 5 y 6). Se reiniciaron, posteriormente, los estiramientos del cuádriceps, consiguiendo incremento del volumen y fuerza, así como la desaparición de los síntomas. Tres meses más tarde realizaba marcha y carrera normales. Cinco años más tarde la paciente permanecía asintomática con marcha y ejes de las EEII normales. Realizaba ejercicio físico normal.

Quince años después de la cirugía la paciente refería quejas por dolor en la zona anteroexterna de la rodilla derecha tras deambulación prolongada, subir y bajar escaleras así como en sedestación prolongada. Se diagnosticó de síndrome de hiperpresión rotuliana externa. Presentaba rótulas centradas en reposo y desplazamiento de las rótulas por el surco troclear centrado. Se evidenciaba hiperpresión rotuliana

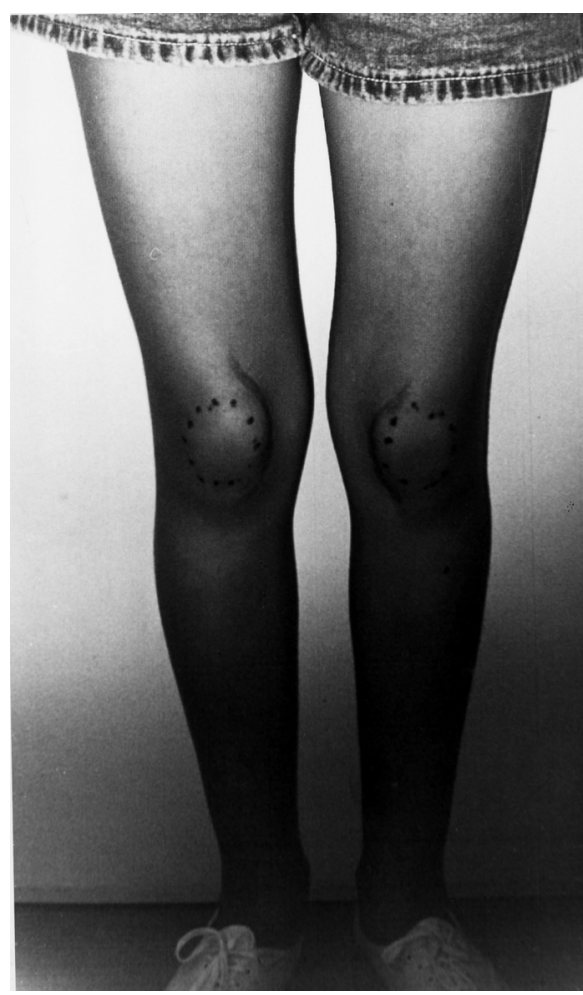

Figura 6. Aspecto postoperatorio de las EEII. Se observa la alineación correcta de las rótulas. 

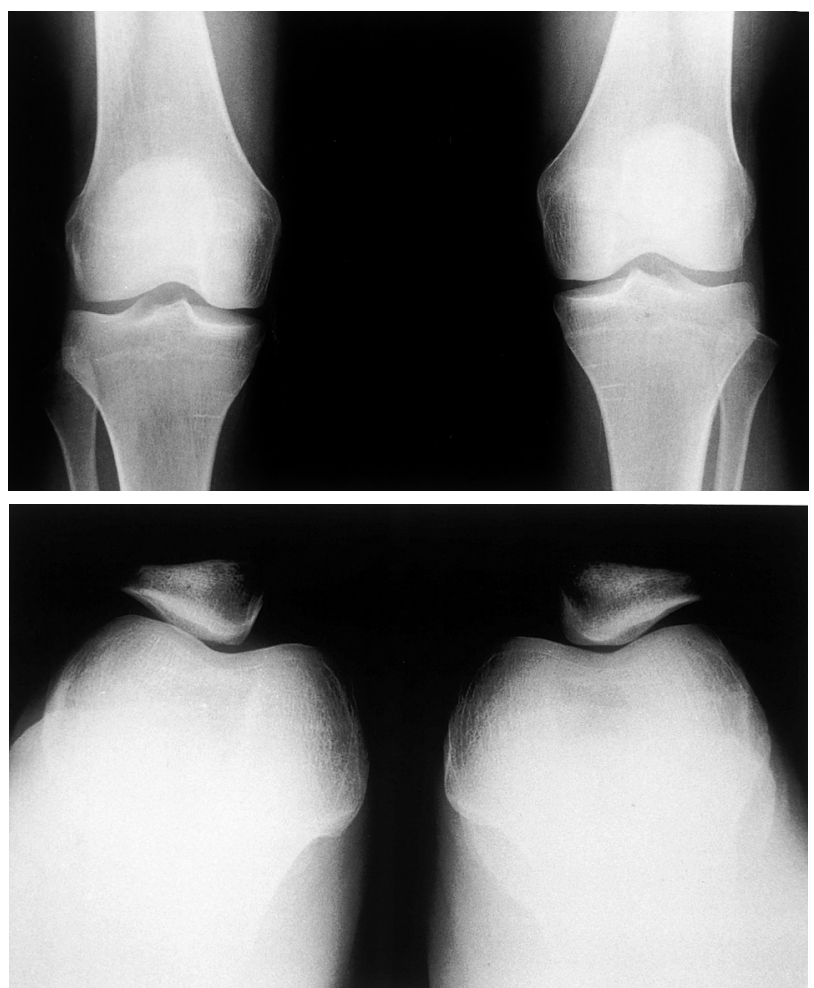

Figuras 7a y 7b. Aspecto radiológico quince años después de la cirugía. Obsérvese la posición centrada de las rótulas, así como displasia de las rótulas (tipo Wiberg III).

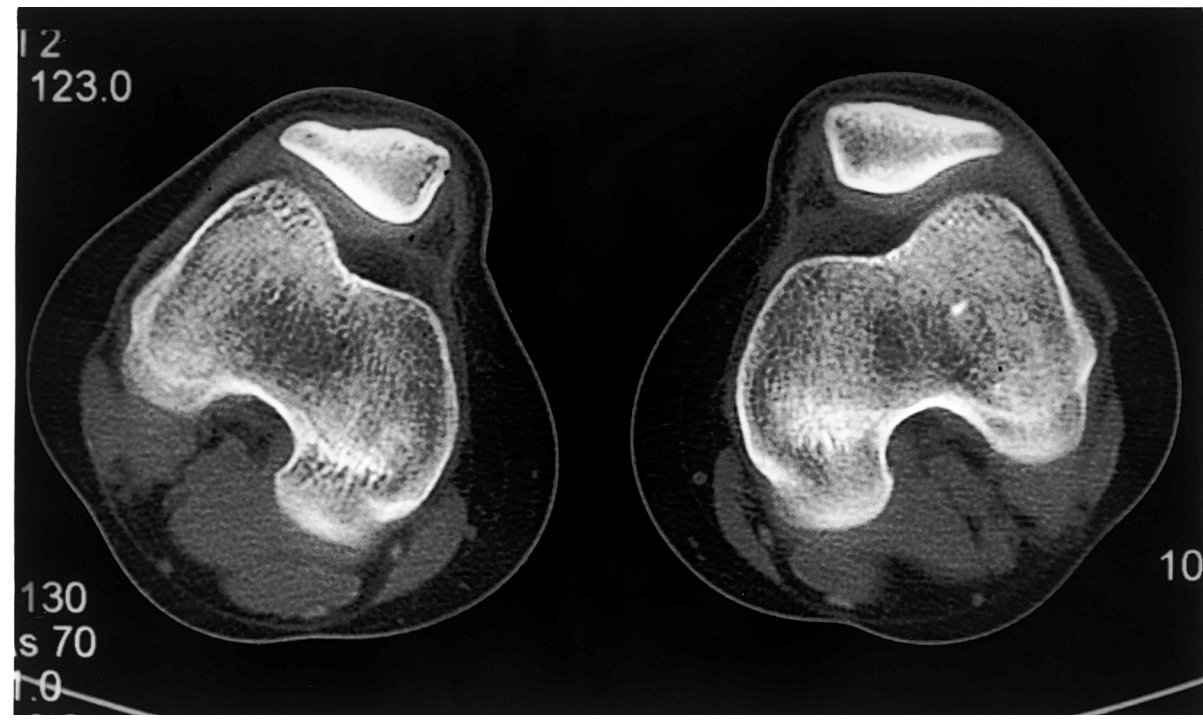

Figura 8. TAC realizado quince años después de la cirugía que muestra hipoplasia del cóndilo medial femoral. 
externa con báscula rotuliana (Figs. 7 a y 7 b). La TAC mostraba (Fig. 8) hipoplasia del cóndilo femoral medial, ya sospechado por presentar cóndilo medial menos prominente en la exploración física de la rodilla. La RNM mostraba condropatía grado I-II de la faceta externa de la rótula derecha.

\section{DISCUSIÓN}

Eppley ${ }^{7}$, Millar y col ${ }^{11}$ y Shellock y col ${ }^{12}$ describieron subluxaciones mediales tras fracasos de liberación retinacular externa. Shellock y col ${ }^{12}$ encontraron una incidencia del $40 \%$ de subluxaciones en las rótulas contralaterales, no intervenidas, en los pacientes con liberación retinacular externa. Por tanto, la desalineación podría ser bilateral y de ello podemos deducir que la subluxación medial existe antes incluso de realizar la liberación retinacular externa, pero que no es detectada por medios convencionales. Esos autores describen la técnica de la RNM dinámica (en los primeros $30^{\circ}$ de flexión) como técnica diagnóstica. En el caso que presentamos no se realizó TAC ni RNM al principio debido a que la inspección y exploración clínica hacían evidente el diagnóstico. Además, la ansiedad de los padres en recibir una pronta solución, por el dolor fémoro-rotuliano y la imposibilidad física para la deambulación de la chica, nos indujo a realizar el tratamiento quirúrgico sin dilación.

Patomecánica. Existen diversos factores que ayudan a explicar la inestabilidad rotuliana del caso que presentamos:

Una pronación excesiva durante la deambulación causa alteraciones en la mecánica fémoro-rotuliana que es reforzada por el varo tibial. Si se le añade una anteversión femoral excesiva (AFE) sin compensación distal (ausencia de TTE compensadora, como ocurría en el caso que presentamos), ni proximal (ángulo $\mathrm{Q}$ normal), entonces puede explicarse la fisiopatología de la inestabilidad rotuliana medial.

La displasia rotuliana (Wiberg tipo III) junto con la hipoplasia condílea femoral medial facilitan la dirección medial de la inestabilidad (la inestabilidad externa se asocia con hipoplasia del cóndilo lateral).

La sincronía de la contracción del vastus medialis obliquus en relación a otros músculos, especialmente el vastus lateralis, es fundamental y puede ser anómala en situaciones tales como la desalineación ${ }^{13}$. El dolorimiento de las estructuras laterales inflamadas y la inestabilidad en sí misma producen amiotrofia por inhibición refleja propioceptiva, sobre todo del vastus lateralis, y de esa forma se incrementa la inestabilidad medial. Esa inestabilidad produce dolor fémoro-rotuliano, bloqueos y aprensión que, con la progresiva incapacidad de la paciente para la deambulación provoca mayor amiotrofia y ésta, mayor inestabilidad, cerrándose así el círculo vicioso. La atrofia muscular progresiva puede llegar a agravar una inestabilidad latente o incrementar su frecuencia de episodios de inestabilidad. Imbert y col lo denominan síndrome de etiología múltiple (amiotrofia de algún grupo muscular, y en menor grado de otros grupos, inestabilidad subjetiva y objetiva, alteraciones propioceptivas, dolorimiento local, alteraciones rotacionales, etc), en el cual la exploración física es básica en orden a obtener la información ${ }^{14}$.

El retináculo lateral para-rotuliano es el principal estabilizador pasivo externo. Por la desalineación crónica, en la inestabilidad medial ocurre lo contrario a la situación habitual (de inestabilidad externa) debido a que el tope del ligamento fémororotuliano externo es laxo y se encuentra retraído el medial.

Los defectos de alineación de los ejes de la rodilla, cuando son severos, pueden originar una inestabilidad basada en la dificultad del sistema muscular para asegurar la marcha estable cuando el brazo de acción está desequilibrado. También provocan que el deslizamiento de la rótula sobre el surco femoral sea anómalo. En tal caso las fuerzas que se requieren para el control de la rótula pueden ser superiores al poder de ciertos grupos musculares cuya acción es interrumpida momentánea o temporalmente. Tras un período de tiempo variable se observa una cierta laxitud de determinadas estructuras que hacen incrementarse la deformidad y la inestabilidad. No existe duda de que la evolución años más tarde es hacia los cambios degenerativos artrósicos. 


\section{Síndrome de desalineación torsional severa}

Ya Sommerville ${ }^{15}$ explicaba que la marcha de los pacientes con AFE muestra rótulas convergentes y es, con frecuencia, una marcha torpe con la característica de que los repetidos intentos de RE provocan TTE compensadora. Denomina a esta anomalía rotacional "persistent fetal alignment of the hip" ("alineación fetal persistente de la cadera"). Fue el primero en realizar osteotomías de realineación, en ocho pacientes con alteraciones severas torsionales. Swanson ${ }^{16}$ describió que la AFE puede causar deformidades del desarrollo debido a las fuerzas torsionales que recibe perpendicularmente el cartílago de crecimiento, lo cual explicaría la asimetría y unilateralidad que ocurre en ciertos casos.

James $^{1}$ describió pacientes con trastornos rotulianos secundarios a alteraciones en la alineación de las EEII in atletas activos, con dolor persistente de rodilla, patella alta, recurvatum y ángulo Q aumentado, que denominó "miserably malalignment syndrome", similar a lo descrito por Meister y James ${ }^{6}$. Este autor describió el espectro de desalineación de la AFE, TTE, tibias varas, rótulas convergentes bilaterales ("rótulas bizcas") y consideraba desalineación rotacional severa la que afecta a los pacientes con AFE y dolor anterior de rodilla secundario a alteración de la biomecánica fémororotuliana. Delgado y $\mathrm{col}^{5}$ consideran como severos aquellos casos con desalineación que desarrollan dolor rotuliano. Nuestro criterio incluye: AFE, desalineación tibial (genu varum y/o TTE), dolor fémoro-rotuliano y/o inestabilidad rotuliana con afectación funcional de la marcha.

Eppley $^{7}$ describió las siguientes características en los pacientes afectos de subluxaciones mediales iatrogénicas: dolor fémoro-rotuliano persistente que no responde al tratamiento (estiramientos del cuádriceps), AFE, TTE, rótulas convergentes o "bizcas", rótulas que se subluxan con facilidad en dirección medial, radiografía normal y ángulo Q normal. Nuestra paciente poseía todas esas características excepto la torsión tibial externa.
Cooke ${ }^{17}$ también describió su experiencia con este tipo de anomalías y resalta su buen pronóstico tras la cirugía. Todos esos autores se refieren al morfotipo de la AFE con TTE compensadora que puede estar asociado o no con "genu varum". Es conocido que este morfotipo se asocia, en algunos casos, con sintomatología fémororotuliana en forma de síndrome rotuliano o inestabilidad rotuliana externa. Hasta el momento no se ha asociado con inestabilidad medial rotuliana.

El síndrome de desalineación severo no debe ser confundido con las desalineaciones no severas que se ven en consulta frecuentemente, en la que la AFE no provoca repercusión clínica alguna, no hay inestabilidad rotuliana ni afectación funcional de la marcha. De hecho el 30\% de los adolescentes que presentan AFE también presentan TTE (suele ser compensadora) y no deben ser tratados ${ }^{18,19}$.

\section{Tratamiento del síndrome de desalineación torsional severa}

La desalineación torsional provoca un anómalo eje de movilidad articular en relación con la línea de progresión con lo que la rótula realiza un recorrido curvilíneo desde la flexión a la extensión. La desalineación y la inestabilidad fémoro-rotuliana rara vez requieren tratamiento fuera de la rodilla. La indicación quirúrgica en pacientes con desalineación torsional severa es excepcional, preconizándose sólo si existe alteración funcional de la marcha y/o síndrome rotuliano doloroso persistente $\mathrm{e}^{17}$, lo cual es aún más raro en niños. Pero en los raros casos de síndrome de desalineación torsional severa el tratamiento quirúrgico se realiza por medio de alineación rotuliana y osteotomía desrotatoria asociada. Con las osteotomías rotacionales (con frecuencia tibial y femoral) se consigue corregir la deformidad y eliminar el dolor 5 .

También Staheli ${ }^{20}$ afirma que aunque el tratamiento quirúrgico está rara vez indicado, las osteotomías a doble nivel son efectivas porque eliminan el dolor rotuliano. Este autor recomienda tratamiento quirúrgico de la AFE con RI de la cadera $>85^{\circ}$ y $\mathrm{RE}<10^{\mathrm{o} 19}$. La osteotomía desrotatoria tibial es recomendada para torsión de 
$\geq 30^{\circ}$. Mosca y Staheli reconocen la necesidad de osteotomías a doble nivel en casos severos $^{21}$. También incluyen la cosmética como indicación primaria. Para nosotros la cosmética no es criterio de tratamiento suficiente.

Bruce y Stevens ${ }^{4}$ trataron quirúrgicamente 14 pacientes con "miserable malalignment syndrome" (AFE y TTE excesiva) asociado a dolor fémoro-rotuliano significativo pero sin inestabilidad. Realizaron osteotomía femoral ipsilateral desrotatoria (externa) y osteotomía desrotatoria tibial (interna). La sección o liberación retinacular lateral se realizó en los pacientes con inclinación rotuliana pasiva negativa.

En los casos de síndrome de desalineación torsional severa con afectación rotuliana y funcional se han descrito diversos tipos de osteotomías: osteotomía distal femoral distal femoral (RE) con osteotomía proximal tibial $(\mathrm{RI})^{5}$; osteotomía femoral subtrocantérea ${ }^{21}$; osteotomía proximal tibial desrotatoria valguizante ${ }^{17}$; o una osteotomía desrotatoria tibial aislada supratuberositaria ${ }^{6}$. Tan sólo Cooke y col realizan liberación retinacular externa asociada $^{17}$. La alineación mediante osteotomías óseas es obligatoria en las alteraciones rotacionales severas ya que la alineación rotuliana aislada puede no ser suficiente. Así, Flandry y Hughston ${ }^{22}$ demostraron una alta tasa de fracasos en la realineación del aparato extensor de la rodilla cuando la causa subyacente del dolor femoro-rotuliano era una AFE, TTE o ambos.

En el caso que presentamos, la ausencia de TTE compensadora podría haber sido uno de los factores causantes de la excepcional dirección medial de la inestabilidad. La osteotomía femoral proximal desrotatoria era la única alternativa para poder realinear el mecanismo extensor (centraje de la rótula sobre el surco troclear) y con ello corregir el deslizamiento anómalo de la rótula. El recentraje retinacular en este caso fue necesario por la tendencia hacia la posición medial de la rótula debido a la cronicidad (desarrolló una retracción del retináculo medial y laxitud del lateral).

La plicatura retinacular externa no es un procedimiento inocuo. La presencia de un síndrome de hiperpresión rotuliana externa a largo plazo en nuestra paciente nos hace cuestionarnos la necesidad de esa liberación como gesto asociado debido a que la báscula rotuliana lateral tiene un efecto destructivo sobre el cartílago articular. Pensamos que la osteotomía femoral proximal desrotatoria aislada pudiera haber sido suficiente.

Concluimos que la subluxación recidivante medial de rótula es una manifestación no descrita del síndrome de desalineación torsional severa de las EEII como consecuencia de la ausencia de TTE compensadora, e hipoplasia del cóndilo femoral medial. Al evaluar las inestabilidades rotulianas asociadas con alteraciones torsionales, debe buscarse la confirmación dinámica para determinar que la inestabilidad es externa y no medial. En caso de incapacidad severa para deambular normalmente la corrección requiere la realineación femoral proximal además de la realineación rotuliana.

\section{BIBLIOGRAFÍA}

1. JAMES SL. Chondromalacia of the patella in the adolescent. En: Kennedy JC. The injured adolescent knee. Baltimore: Williams and Wilkins, 1979, 205-251.

2. Salenius P, VAnKa E. The development of the tibiofemoral angle in children. J Bone Joint Surg Am 1975; 57: 259-261.

3. Cahuzac JPH, Vardon D, Sales de Gauzy J. Development of the clinical tibiofemoral angle in normal adolescents. J Bone Joint Surg 1995; 77-B, 5: 729-732.

4. BRuce WD, StEVEns PM. Surgical correction of miserable malalignement syndrome. J Pediatr Orthop 2004, 24: 392-396.

5. Delgado DE, Schoenecker PL, Rich MM, CAPELLI AM. Treatment of severe torsional malalignment syndrome. J Pediatr Orthop 1996; 4: 484-488.

6. Meister K, JAmes SL. Proximal tibial derotation osteotomy for anterior knee pain in the miserably malaligned extremity. Am J Orthop 1995; 2: 149-155.

7. EPPLEY RA. Medial patellar subluxation. En: Fox JM, Del Pizzo W. The patellofemoral joint. New York: McGraw-Hill, 9: 1993, 149156. 
8. FULKERSON JP, HuNGERFORD DS. Disorders of the patellofemoral joint. Baltimore: Williams and Wilkins, Second edition, 1990.

9. Hughston JC, DEESE M. Medial subluxation of the patella as a complication of lateral retinacular release. Am J Sports Med 1988; 16, 4: 383-388.

10. Hughston JC, Flandry F, Brinker MR, TERry GC, Mills JC. Surgical correction of medial subluxation of the patella. Am J Sports Med 1996; 24: 486-491.

11. Miller PR, Klein RM, Teitge RA. Medial dislocation of the patella. Skeletal Radiol 1991; 20: 429-431.

12. Shellock FG, Mink JH, Deutsch A, Fox JM, FERKEL RD. Evaluation of patients with persistent symptoms following lateral retinacular release by kinematic MRI of the patellofemoral joint. Arthroscopy 1990; 6: 226.

13. GRESAMER RP. Current concepts review. Patellar malalignment. J Bone Joint Surg Am 2000; 82: 1639-1650.

14. IMBERT JC, BRUNET-GuEdJ E. Instabilités du genou. Editions Techniques. Encycl. Méd. Chir. (Paris-France). Appareil locomoteur, 14080 B10, 1992.
15. SommervilLe EW. Persistent foetal alignement of the hip. J Bone Joint Surg Br 1957; 39, 1: 106-113.

16. Swanson AB, Greene PW, Allis HD. Rotational deformities of the lower extremity in children and their clinical significance. Clin Orthop 1963; 27: 157-175.

17. Cooke TDV, Price N, Fisher B, Hedden D. The inwardly pointing knee. An unrecognized problem of external rotational malalignment. Clin Orthop 1990; 260: 56-60.

18. FABRY G, MACEWEN GD, SHANDS AR. Torsion of the femur - a study in normal and abnormal conditions. J Bone Joint Surg Am 1973; 55: 1726-1738.

19. STAHELI LT. Torsion-treatment indications. Clin Orthop 1989; 247: 61-66.

20. STAHELI LT. Rotational problems in children (AAOS Instructional Course Lectures). J Bone Joint Surg Am 1993; 75-A: 939-949.

21. Mosca VS, STAHeli LT. Surgical management of torsional and angular deformities of the lower extremities. En: Chapman M. Operative Orthopedics, vol 3. Philadelphia: Lippincot, 1988.

22. Flandry F, Hughston JC. Complications of extensor mechanism surgery for patellar malalignment. Am J Orthop 1995, 24: 534543. 